\title{
Un análisis de la relación entre ambiente familiar y delincuencia juvenil
}

\author{
LOURDES MIRÓN REDONDO, ANGELES \\ LUENGO MARTÍN, JORGE SOBRAL FERNÁNDEZ \\ y JOSÉ M. OTERO LÓPEZ
}

Universidad de Santiago

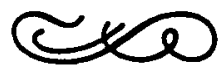

\section{Resumen}

En este trabajo se analiza la relación entre las interacciones afectivas y normativas del ambiente familiar, $y$ la conducta delictiva de los adolescentes varones. La muestra del estudio estaba compuesta por tres grupos de adolescentes: no delincuentes, delincuentes no detectados y delincuentes detectados (institucionalizados). Los resultados de los análisis realizados ponen de manifiesto la existencia de diferencias significativas entre las caracteristicas familiares de delincuentes y no delincuentes. Concretamente, los datos del estudio permiten afirmar que: 1) las variables afectivas parecen más importantes que las referidas a los aspectos normativos para discriminar entre los grupos de sujetos, y 2) entre las variables afectivas serian el apego del hijo hacia los padres y el conflicto familiar las que aparecen como especialmente relevantes para discriminar entre delincuentes y no delincuentes, asociándose un alto nivel de apego/bajo nivel de conflicto con no delincuencia, y un bajo nivel de apego/alto nivel de conflicto con delincuencia.

\section{Abstract}

This paper examines the relationship between affective and normative interacctions of $f a-$ mily enviroment, and male adolescent delictive behavoir. Sample of study was compossed by 3 groups of adolescents: non-delinquents, non-detected delinquents, and detected delinquents (institucionalized). Results of realized analysis point out the existence of significative diferences between family characteristics of delinquents and non-delinquents. Concretly, data of the study permit to affirm that: 1) affective variables seem more important than those refered to normative apects to discriminating among the groups of subjects, and 2) among the affective variables would be the attachment of the son toward his parents and family conflict those that appear as specially relevant to discriminanting between delinquents and non-delinquents, associating bigh level of attachment/low level of conflict with non-delinquency, and low level of attachment/bigh level of conflict with delinquency.

Dirección del autor: Departamento de Psicología General. Facultad de Filosofía y Ciencias de la Educación. Universidad de Santiago de Compostela. 


\section{INTRODUCCION}

El interés por el estudio de la familia en relación con la delincuencia juvenil se remonta a las primeras investigaciones en el área. Sin embargo, los factores familiares analizados han ido cambiando a lo largo del tiempo.

En un primer momento se prestaba una atención prioritaria a las variables referidas a la estructura familiar, tales como el tamaño de la familia o el hogar roto; mientras que, en la actualidad, predominan los estudios centrados en las variables de funcionamiento familiar. Este cambio en las variables familiares analizadas en los estudios de delincuencia juvenil se debe a que la relación encontrada entre familia y delincuencia sólo puede explicarse, como se ha demostrado empíricamente (Hirschi, 1969; Rutter, 1971; Chilton y Markle, 1972), recurriendo al análisis del funcionamiento familiar. Es decir, los hogares con muchos miembros, o en los que uno o ambos padres están ausentes, llevan asociadas, generalmente, una serie de características desfavorables, tales como falta de atención, conflicto, pobres interacciones afectivas y/o inadecuada supervisión, que serían las responsables de la conducta delictiva del hijo (Spicer y Hampe, 1975; Offord, 1982; McCord, 1982, 1983; Fischer, 1984).

\section{ESTADO ACTUAL DE LA LITERATURA}

La influencia del entorno familiar en la socialización de los hijos ha sido analizada, fundamentalmente, en función de las variaciones en: 1) las relaciones afectivas entre padres e hijos, y 2) la supervisión o el control de los padres sobre la conducta del hijo.

\section{Relaciones afectivas familiares y delincuencia juvenil}

Las relaciones afectivas entre padres e hijos son fundamentales para el desarrollo adecuado del individuo. Por ello, se sugiere que la ausencia de tales vínculos incrementará, de manera importante, la probabiliad de que el hijo se involucre en actividades delictivas (Linden y Fillmore, 1981; Linden y Hackler, 1973).

Esta relación entre falta de apego a la familia y delincuencia estaría fundamentada, a nivel teórico en las Teorías de Control Social (Nye, 1957, 1958; Hirschi, 1969).

Hirschi (1969) considera que el apego entre padres e hijos permite a los padres controlar, de manera directa e indirecta, la conducta del hijo, recibir información acerca de sus actividades y establecer relaciones de afecto y respeto que favorezcan el proceso de socialización. La ausencia de apego elimina la posibilidad de que los padres establezcan controles, con lo cual el proceso de socialización se dificulta, y aumenta la probabilidad de que el hijo desarrolle una conducta delictiva.

Los resultados de diversas investigaciones confirman que la inexistencia de vínculos afectivos y/o la presencia del conflicto en las relaciones entre padres e hijos se asocian con la delincuencia del adolescente (Reiss, 1971; Linden y Hackler, 1973; Hanson y col., 1984; Loeber y Dishion, 1981). 
A un nivel más específico, los estudios acerca de la relación entre las interacciones afectivas en el hogar y la delincuencia juvenil, se han ocupado, fundamentalmente de las interacciones entre el padre y la madre, y entre el adolescente y cada uno de sus padres.

La inexistencia de una adecuada relación afectiva entre el padre y la madre es importante, no sólo porque la ausencia de apego entre ellos genera conflicto, y el conflicto incrementa la probabilidad de delincuencia (Norland y col., 1979), sino también porque el desacuerdo generado por el conflicto debilita su autoridad de cara a los hijos (Davies y Sinclair, 1971), haciendo más difícil la tarea de socialización (Bebchuck, 1983).

$\mathrm{La}$ investigación sobre este punto ha confirmado la existencia de una relación clara entre conflicto parental y delincuencia del adolescente. Tal relación se ha encontrado tanto en estudios transversales como longitudinales, y tanto en trabajos basados en autoinformes del adolescente como en aquellos en los que la información se recoge de los padres.

Glueck y Glueck $(1950,1968)$, comparando un grupo de adolescentes no delincuentes con otro de delincuentes detectados (oficiales), encuentran que los adolescentes delincuentes informan de unas relaciones significativamente menos afectuosas entre sus padres que los adolescentes no delincuentes. Norland y col. (1979) y Reiss (1975) señalan la existencia de estas mismas diferencias comparando delincuentes no detectados con no delincuentes; y Empey y Lubeck (1971a) confirman estos hallazgos en un estudio en el que utilizan ambos tipos de delincuencia (detectada y no detectada).

Del mismo modo, el trabajo de Gove y Crutchfield (1982), realizado en base a la respuestas de los padres a entrevistas sistemáticas, evidencia que las características del matrimonio de los padres juegan un papel importante en la aparición de la conducta delictiva de los adolescentes varones, en el sentido de que unas relaciones maritales poco afectuosas correlacionan con delincuencia del hijo.

Los estudios longitudinales encuentran resultados muy similares, proporcionando mayor validez a los hallazgos de las investigaciones transversales. Así, West (1969), Farrington (1973) y Farrington y West (1971), trabajando con datos recogidos de los adolescentes y sus padres durante un período de catorce años, concluyen que una de las principales características de las familias de los adolescentes que desarrollan conductas delictivas es la ausencia de armonía en las relaciones entre los padres.

En cuanto a las relaciones afectivas entre el hijo y sus padres, tradicionalmente, se ha asignado un papel crucial a las relaciones afectivas con la madre en el proceso de desarrollo de los hijos. Por ello, la hipótesis de que la ausencia de vinculación afectiva con la madre tiene un importante efecto sobre la conducta desviada del hijo es uno de los puntos de partida de algunas investigaciones en el área de la delincuencia juvenil. Los resultados de estas investigaciones muestran que el rechazo mutuo entre la madre y el hijo se asocia fuertemente con la conducta delictiva del adolescente. De nuevo, este hallazgo aparece tanto en estudios transversales realizados en base a autoinformes del adolescente (Glueck y Glueck, 1950; Hirschi, 1969), como en aquellos en los que se recogen los datos de los padres (Olweus, 1980; Harbin y Madden, 1983). Los hallazgos del estudio longitudinal llevado a cabo por McCord (1979) recogiendo información a lo largo 
de cinco años, mediante la observación directa de la conducta de padres e hijos en el hogar, confirman que el rechazo de la madre hacia el hijo, y la ausencia de confianza entre ambos es uno de los principales predictores de la conducta delictiva del adolescente.

Debido al papel predominante atribuido a las relaciones afectivas entre la madre y el hijo, algunas investigaciones han obviado el análisis de los vínculos afectivos padre-hijo de cara a la aparición de conducta delictiva en el adolescente. Sin embargo, los resultado de las investigaciones que han tenido en cuenta esta variable muestran que el impacto de la falta de apego entre padre e hijo sobre la delincuencia del joven es similar al de la ausencia de relaciones afectivas con la madre (Nye, 1958; Hirschi, 1969; Glueck y Glueck, 1950; West y Farrington, 1977).

La conclusión general de los estudios acerca de la relación entre vínculos afectivos familiares y delincuencia juvenil sería entonces; que la existencia de unas relaciones afectivas adecuadas entre ambos padres y entre éstos y el hijo se asocia con ausencia de delincuencia, mientras que el conflicto y/o las relaciones no afectuosas entre padres e hijos se asocian con conducta delictiva del adolescente.

\section{Supervisión parental y delincuencia juvenil}

El otro aspecto de la socialización de los hijos primordialmente analizado en los estudios sobre familia y delincuencia juvenil es el control (supervisión) ejercido por los padres sobre la conducta de los hijos.

La supervisión paterna es uno de los mecanismos a través de los cuales los padres promueven la internalización de normas en los hijos. La internalización de normas es esencial para que el individuo se integre adecuadamente en las sociedad, dado que si el sujeto no internaliza las normas sociales, cuando no esté sometido a un control externo no contará con ningún tipo de control interno que inhiba su conducta.

Los estudios que han analizado la importancia de la supervisión parental en la conducta delictiva del adolescente encuentran, consistemente, que una supervisión débil o excesivamente rígida se relaciona con la conducta desviada.

La mayor parte de estas investigaciones han encontrado relación entre supervisión paterna débil o escasa y delincuencia (Fischer, 1983). Así, Glueck y Glueck (1970) trabajando con un grupo de delincuentes detectados y otro de no delincuentes, encuentran que una de las variables más importantes y significativas para establecer diferencias entre ambos grupos es la supervisión parental, siendo ésta significativamente menor en el grupo de delincuentes. En este mismo sentido, autores como Stanfield (1966), Hischi (1969), Cortés y Gatti (1972), West y Farrington (1977) y Wilson (1980) señalan que los padres de los adolescentes delincuentes tienen, generalmente, escaso control e información acerca de las actividades de sus hijos. Del mismo modo, Goldstein (1984), en un estudio realizado con datos recogidos en la familia y la escuela, encuentra que la escasa o insuficiente supervisión paterna es el «factor crucial" con respecto al desarrollo de conducta delictiva por parte de los adolescentes. 
McCord (1979) confirma que la supervisión débil durante la infancia es uno de los principales predictores de la criminalidad posterior.

Algunos otros estudios han encontrado, sin embargo, relación entre supervisión excesivamente rígida y delincuencia. En este sentido, Kogan (1980) afirma que las familias de adolescentes delincuentes suelen estar excesivamente orientadas hacia el control, intentando, incluso, incrementar el nivel de cohesión familiar (generalmente bajo) aumentando el grado de control ejercido sobre los hijos. Kogan considera que este énfasis excesivo en el control, sobre todo en la adolescencia puede generar conflicto en las relaciones entre padres e hijos, incrementando, de este modo, la probabilidad de la conducta delictiva del adolescente.

Glueck y Glueck (1968) trabajando con autoinformes de adolescentes, encuentran evidencia de que tanto la supervisión paterna escasa como la excesivamente estricta se relacionan con conducta desviada del hijo. Conger (1977) confirma estos resultados en un estudio realizado en base a datos recogidos de los padres. Por último, el trabajo de Wright (1982) realizado para determinar la relación entre la conducta desviada de los adolescentes y la edad en la que los padres les permiten una mayor autonomía (reduciendo la supervisión), muestra que la conducta desviada es más probable entre aquellos jóvenes que o bien disponen de libertad a edades tempranas, o bien están excesivamente controlados durante la fsase intermedia de la adolescencia (alrededor de los quince años). Es decir, aunque existe mayor evidencia empírica de la relación entre ausencia de supervisión y delincuencia, la supervisión muy estricta o el control excesivo, especialmente en la adolescencia, se han relacionado también empíricamente con la conducta delictiva del hijo.

En general, la literatura revisada sugiere que tanto los vínculos afectivos entre padres e hijos, como el grado de supervisión de los padres sobre la conducta del hijo, juegan un papel fundamental en la conducta delictiva del adolescente.

Partiendo de estos hallazgos, en el presente estudio analizamos la relación entre las interacciones familiares y la delincuencia del hijo, con el objetivo de determinar cuáles, de entre los aspectos afectivos y normativos señalados como relevantes en las investigaciones previas, pueden ser más importantes en la comprensión de la conducta delictiva de lo adolescentes varones.

\section{ESTUDIO}

\section{Muestra}

La muestra del estudio estaba compuesta, inicialmente, por 347 adolescentes varones, de edades comprendidas entre los doce y los dieciséis años. De ellos, 276 vivían con sus padres, y 71 estaban institucionalizados en Centros de Menores debido a la realización de actividades delictivas.

Con el grupo de adolescentes que vivían en el hogar, y en función de sus autoinformes de conducta antisocial, se formaron dos subgrupos de sujetos. El primero de ellos quedó compuesto por 43 adolescentes que manifestaron no haber realizado ninguna actividad delictiva, y fue utilizado 
en los análisis como grupo control. El segundo subgrupo quedó formado por 47 adolescentes que informaron de la realización de actividades claramente delictivas así como de conductas contra las normas.

El grupo de adolescentes institucionalizados constituía la práctica totalidad de los sujetos, entre los doce y los dieciséis años internados en los Centros de Menores de Galicia (Centro de Palavea, en la Coruña; Centro Santo Anxo, en Lugo, y Centro Divino Redentor, en Orense), en régimen de reforma. De este grupo se eliminaron algunos sujetos debido a sus respuestas erróneas o incompletas a los autoinformes que se les aplicaron.

Así, la muestra utilizada quedó compuesta por tres grupos, diferenciados en cuanto a la variable delincuencia:

- GRUPO 1: No delincuentes $(n=43)$.

- GRUPO 2: Delincuentes no detectados $(n=47)$.

- GRUPO 3: Delincuentes detectados $(n=43)$.

El nivel socioeconómico de la muestra se ha determinado en base a las respuestas de los adolescentes a una serie de ítems referidos a la profesión de los padres, al nivel de estudios de los mismos, y a las características de la vivienda familiar (número de habitaciones en relación con el número de personas que viven en el hogar). Utilizando este índice combinado se ha encontrado (Tabla I) que mientras los no delincuentes y los delincuentes no detectados presentan características socioeconómicas similares, y pertenecen, la mayoría, a la clase media, los delincuentes detectados (institucionalizados) pertenecen casi exclusicamente a la clase baja. Este dato puede interpretarse en la línea de los resultados de las investigaciones que encuentran que lo que correlaciona con clase baja es la institucionalización y no la conducta delictiva en sí misma (Farrington, 1979).

TABLA I

Número y porcentaje de sujetos de cada grupo pertenecientes a cada una de las clases sociales

\begin{tabular}{|c|c|c|c|c|c|c|}
\hline \multirow[b]{3}{*}{ Clase social } & \multirow{2}{*}{\multicolumn{2}{|c|}{$\begin{array}{c}\text { Grupo } 1 \\
\begin{array}{c}\text { No } \\
\text { delincuentes }\end{array}\end{array}$}} & \multirow{2}{*}{\multicolumn{2}{|c|}{$\begin{array}{c}\text { Grupo } 2 \\
\text { Delincuentes } \\
\text { no detectados }\end{array}$}} & \multirow{2}{*}{\multicolumn{2}{|c|}{$\begin{array}{c}\text { Grupo } 3 \\
\begin{array}{c}\text { Delincuentes } \\
\text { detectados }\end{array}\end{array}$}} \\
\hline & & & & & & \\
\hline & n. ${ }^{\circ}$ & $\%$ & n..$^{\circ}$ & $\%$ & n. ${ }^{\circ}$ & $\%$ \\
\hline $\begin{array}{l}\text { Baja } \\
\text { Media-baja } \\
\text { Media-alta }\end{array}$ & $\begin{array}{l}11 \\
19 \\
12\end{array}$ & $\begin{array}{l}26.2 \\
45.2 \\
28.6\end{array}$ & $\begin{array}{l}11 \\
22 \\
14\end{array}$ & $\begin{array}{l}23.4 \\
46.8 \\
29.8\end{array}$ & $\begin{array}{r}37 \\
2 \\
0\end{array}$ & $\begin{array}{c}94.9 \\
5.1 \\
0\end{array}$ \\
\hline
\end{tabular}

\section{Medición de Variables}

En el presente estudio se consideró como variable dependiente la conducta delictiva del adolescente. Para operacionalizarla se ha utilizado la Escala de Conducta Antisocial desarrollada por Mateo (1982). En un trabajo posterior (Núnez, 1983) se introdujeron algunas modificaciones en el instrumento elaborado por Mateo: se limitó el número de ítems, se cambió el 
sistema dicotómico de respuesta a los ítems (si/no) por otro que tiene en cuenta la frecuencia de realización de cada una de las actividades (nunca, una o dos veces, de dos a seis veces, a menudo); y se realizó un Análisis Factorial de los ítems en el que se obtuvieron dos factores de segundo orden. El primero de estos factores engloba 24 items referidos a conductas de agresión y robo (es decir, conductas ilegales). El segundo factor quedó compuesto por 12 ítems referidos a conductas antisociales más leves, que no tienen carácter ilegal.

La Escala de Conducta Antisocial se ha utilizado en el presente estudio, fundamentalmente, para establecer dos grupos entre los adolescentes que vivían con sus padres: no delincuentes y delincuentes no detectados. Se clasificó como no delincuentes a los adolescentes que o bien no habían realizado nunca ninguna de las actividades recogidas en la Escala, o bien habían realizado una o dos veces una conducta contra las normas (del $2 .^{\circ}$ factor). Se consideraron delincuentes no detectados a los sujetos que habían realizado, al menos, 2 conductas del primer factor (ilegales) y varias de las del segundo factor (hasta puntuar un total de 15 en la escala, teniendo en cuenta que a la respuesta "una o dos veces" se le asignó un valor 1 , a "de dos a seis veces" un valor 2 , y a "a menudo" un valor 3 ).

La Escala fue aplicada también al grupo de adolescentes institucionalizados con el objetivo de «validar» el criterio elegido para la clasificación de los delincuentes no detectados. El análisis de las respuestas de este grupo permitió confirmar la idoneidad del criterio, dado que sus informes de conducta delictiva son muy similares a los del grupo de delincuentes no detectados.

Las variables independientes del estudio hacen referencia a las relaciones afectivas y normativas del ambiente familiar del adolescente.

Entre las variables afectivas se incluyen: el apego entre el hijo y sus padres, la cohesión y el conflicto familiar, la «calidad» de las relaciones entre el adolescente y cada uno de sus padres, y el conflicto entre el padre y la madre y entre el adolescente y ambos padres.

$\mathrm{El}$ apego será entendido como «una vinculación afectiva de fuerte intensidad" (Bowlby, 1969, 1973) entre el hijo y sus padres, y será operacionalizado en base al Inventario de Apego a los Padres (Inventory of Parent Attachment) desarrollado por Greenberg y Col. (1983) a partir de la definición de Bowlby. Este inventario consta de tres factores: Confianza, Comunicación y Alienación. La puntuación total de apego se obtiene sumando las puntuaciones del sujeto en los factores de confianza y comunicación, y restando a esta suma la puntuación en el factor de alienación. En el presente estudio se utilizarán como variables las puntuaciones del sujeto en cada uno de los factores, así como la puntuación total de apego.

La cohesión familiar es un concepto muy relacionado con el de apego, que será entendido en este trabajo como el grado de apoyo mutuo, afecto y confianza entre los miembros de la familia (Moos, 1976), y que será operacionalizado en base a la subescala de Cohesión de la Escala de Ambiente Familiar (Family Environment Scale) elaborada por Moos y col. (1981).

El conflicto familiar será entendido como el grado de agresividad y falta de acuerdo entre los miembros de la familia (Moos, 1976), y será operacionalizado en base a la Subescala de Conflicto de la Escala de Ambiente Familiar de Moos y col. (1981). 
Para evaluar más concretamente la calidad de las relaciones entre el hijo y la madre y entre el hijo y el padre, se pidió a los adolescentes que definiesen cada. una de estas relaciones en una escala de respuestas que incluía desde "muy malas» (respuestas a la que se le asignó un valor 1) a "muy buenas» (valor 7). Del mismo modo, el conflicto entre el padre y la madre, y entre el adolescente y ambos padres se evaluó en base a dos ítems; con respecto a los cuales el sujeto debía indicar su grado de acuerdo: 1) «en mi familia hay conflictos entre mis padres», y 2) "yo tengo conflictos con mis padres». Las posibles respuestas incluían desde «muy en desacuerdo» (con un valor asignado de 1) a "muy de acuerdo" (con un valor 7 ).

Las relaciones normativas del ambiente familiar se han analizado en base a dos variables: la organización y el control familiar.

La organización familiar será entendida como el grado de estructuración de las actividades y responsabilidades de los miembros de la familia, y será operacionalizada en base a la Subescala de Organización de la Escala de Ambiente Familiar (Moos y col., 1981).

El control familiar será entendido como el grado de rigidez de la estructura de poder, los roles y los procedimientos utilizados en la familia (Moos, 1976); y será operacionalizado en base a la Subescala de Control de la Escala de Ambiente Familiar (Moos y col., 1981).

De acuerdo con las definiciones de Moos (1976), en este trabajo se considera que un alto grado de organización familiar implicaría una adecuada supervisión de los padres sobre la conducta de los hijos mientras que un alto grado de control implicaría una rigidez excesiva en la supervisión.

Las hipótesis de partida del estudio, formuladas en base a los hallazgos empíricos de las investigaciones precedentes, establecen que, con respecto a las variables familiares afectivas los adolescentes delincuentes, tanto detectados como no detectados, presentarán un menor grado de apego a sus padres (menor confianza y comunicación y mayor alienación), una menor cohesión familiar, y un mayor grado de conflicto en todas las relaciones familiares, que los adolescentes no delincuentes. En cuanto a las variables referidas a los aspectos normativos, se espera que los delincuentes informen de un menor grado de organización y un mayor grado de control que los no delincuentes.

\section{RESULTADOS}

En primer lugar, se realizó un análisis de varianza (utilizando el programa BMDPIV) para comprobar si los tres grupos de adolescentes difieren significativamente en cuanto a las variables familiares analizadas.

Los resultados de este análisis muestran (tal como puede verse en la tabla II) que existen diferencias significativas entre los tres grupos de adolescentes en la práctica totalidad de las variables, con la excepción de la variable relación del hijo de la madre.

En la tabla III aparecen los resultados de la prueba a posteriori de Scheffé y el nivel de significación de las diferencias entre los grupos, tomados dos a dos, para aquellas variables que han resultado significativas en el análisis de varianza. 
TABLA II

Análisis de varianza: Puntuaciones medias, valor $F$ y nivel de significación de las diferencias en las variables entre los 3 grupos de sujetos

\begin{tabular}{|c|c|c|c|c|c|}
\hline & Grupo 1 & Grupo 2 & Grupo 3 & \multirow[b]{2}{*}{$\mathbf{F}$} & \multirow[b]{2}{*}{$\begin{array}{l}\text { Nivel de } \\
\text { significación }\end{array}$} \\
\hline & $\begin{array}{c}\text { No } \\
\text { delincuentes } \\
(n=43) \\
x\end{array}$ & $\begin{array}{c}\text { Delincuentes } \\
\text { no } \\
\text { detectados } \\
(\mathrm{n}=\mathbf{4 7 )} \\
\mathrm{x}\end{array}$ & $\begin{array}{c}\text { Delincuentes } \\
\text { detectados } \\
(n=43) \\
x\end{array}$ & & \\
\hline $\begin{array}{l}\text { Apego } \\
\text { Confianza } \\
\text { Comunicación } \\
\text { Alienación } \\
\text { Cohesión familiar } \\
\text { Conflicto familiar } \\
\text { Conflicto padre-madre } \\
\text { Conflicto hijo-padres } \\
\text { Relación hijo-padre } \\
\text { Relación hijo-madre } \\
\text { Organización } \\
\text { Control }\end{array}$ & $\begin{array}{r}76,27 \\
44,93 \\
43,95 \\
12,27 \\
7,90 \\
1,74 \\
1,88 \\
1,74 \\
6,51 \\
6,62 \\
7,00 \\
3,48\end{array}$ & $\begin{array}{r}43.89 \\
32,70 \\
32,59 \\
21,31 \\
5,42 \\
3,61 \\
3,29 \\
3,21 \\
5,38 \\
5,97 \\
5,70 \\
4,40\end{array}$ & $\begin{array}{r}30,67 \\
29,97 \\
27,93 \\
27,20 \\
4,13 \\
5,55 \\
4,65 \\
4,53 \\
4,51 \\
6,18 \\
5,37 \\
4,39\end{array}$ & $\begin{array}{r}161,12 \\
70,40 \\
86,33 \\
144,35 \\
38,44 \\
47,07 \\
29,20 \\
32,54 \\
17,00 \\
3,74 \\
9,78 \\
4,28\end{array}$ & $\begin{array}{l}, 0000 \\
, 0000 \\
, 0000 \\
, 0000 \\
, 0000 \\
, 0000 \\
, 0000 \\
, 0000 \\
, 0000 \\
\text { N.S. } \\
, 0001 \\
, 01\end{array}$ \\
\hline
\end{tabular}

TABLA III

Valor de la prueba de Scheffé y nivel de significación de las diferencias para la comparación entre los grupos tomados dos a dos

\begin{tabular}{|c|c|c|c|c|c|c|}
\hline & \multicolumn{2}{|c|}{$\begin{array}{l}\text { Comparación } \\
\text { no delincuentes- } \\
\text { delincuentes } \\
\text { no detectados }\end{array}$} & \multicolumn{2}{|c|}{$\begin{array}{l}\text { Comparación } \\
\text { no delincuentes- } \\
\text { delincuentes } \\
\text { detectados }\end{array}$} & \multicolumn{2}{|c|}{$\begin{array}{c}\text { Comparación } \\
\text { delincuentes } \\
\text { no detectados- } \\
\text { delincuentes } \\
\text { detectados }\end{array}$} \\
\hline & $\begin{array}{l}\text { Valor } \\
\text { de } \\
\text { Scheffé }\end{array}$ & $\begin{array}{l}\text { Nivel de } \\
\text { signifi- } \\
\text { cación }\end{array}$ & $\begin{array}{c}\text { Valor } \\
\text { de } \\
\text { Scheffé }\end{array}$ & $\begin{array}{l}\text { Nivel de } \\
\text { signifi- } \\
\text { cación }\end{array}$ & $\begin{array}{l}\text { Valor } \\
\text { de } \\
\text { Scheffé }\end{array}$ & $\begin{array}{l}\text { Nivel de } \\
\text { signifi- } \\
\text { cación }\end{array}$ \\
\hline Apego & $-12,63$ &, 0000 & $-17,41$ &, 0000 & $-5,15$ & .0000 \\
\hline Confianza & $-9,27$ &, 0000 & $-11,10$ &, 0000 & $-2,06$ & N.S. \\
\hline Comunicación & $-9,23$ &, 0000 & $-12,17$ & .0000 & $-3,79$ & .0002 \\
\hline Alienación & 10,43 &, 0000 & 16,85 &, 0000 & 6,79 &, 0000 \\
\hline Cohesión familiar & $-5,80$ &, 0000 & $-8,61$ &, 0000 & $-3,00$ &, 003 \\
\hline Conflicto familiar & 4,68 &, 0000 & 9,70 &, 0000 & 5,04 &, 0000 \\
\hline dre & 4,03 &, 0001 & 7,73 &, 0000 & 3,86 &, 0002 \\
\hline Conflicto hijo-pa- & & & & & & \\
\hline $\begin{array}{l}\text { dres } \\
\text { Relación hijo-padre }\end{array}$ & $\begin{array}{r}4,33 \\
-3,35\end{array}$ &, 0000 & $\begin{array}{r}8,06 \\
-581\end{array}$ & , 0000 & $\begin{array}{r}3,90 \\
-2,58\end{array}$ & ,0002 \\
\hline Organización & $\begin{array}{l}-5,33 \\
-3,39\end{array}$ &, 0009 & $\begin{array}{l}-5,81 \\
-4,17\end{array}$ &, 0001 & $\begin{array}{l}-2,38 \\
-86\end{array}$ & 'N.S. \\
\hline Control & 2,57 &, 01 & 2,49 & 01 &,- 02 & N.S. \\
\hline
\end{tabular}

Los datos de ambas tablas indican que, de acuerdo con lo que se hipotetizaba, los adolescentes delincuentes se diferencian de los no delincuentes en cuanto a las relaciones afectivas y a los aspectos normativos de su entorno familiar. 
Con respecto a las variables afectivas, el grado de apego entre el hijo y los padres se revela como una de las variables fundamentales para diferenciar entre delincuentes y no delincuentes. Las puntuaciones medias, que aparecen en la tabla 2, muestran que son los adolescentes delincuentes los que manifiestan un menor grado de apego hacia sus padres (menor confianza y comunicación y mayor alienación). Los resultados de la prueba a posteriori de Sheffé confirman que los no delincuentes se diferencian significativamente $(p<.0000)$ de ambos grupos de delincuentes en cuanto al apego, tanto considerado globalmente como en cada una de sus dimensiones. Sin embargo, los delincuentes no detectados se diferencian de los delincuentes detectados, al mismo nivel de significación $(p<.0000)$, únicamente en cuanto al apego y a la alineación; y se diferencian a un nivel algo menor $(p<.0002)$ en cuanto a la comunicación; mientras que no aparecen diferencias significativas entre ellos con respecto a la confianza.

La cohesión familiar establece también diferencias significativas entre los grupos, y, de acuerdo con la hipótesis formulada, el nivel de cohesión es menor en las familias de los adolescentes delincuentes. Las mayores diferencias en esta variable se producen entre los no delincuentes y ambos grupos de delincuentes $(\mathrm{p}<.0000)$, siendo algo menor, aunque significativa $(p<.003)$, entre delincuentes detectados y no detectados.

Aparecen también diferencias significativas entre los grupos con respecto al nivel de conflicto familiar, siendo los dos grupos de delincuentes los que presentan los mayores índices de conflicto en el hogar. Sin embargo, esta variable parece importante también para diferenciar entre delincuentes detectados y no detectados, dado que el nivel de significación de las diferencias es idéntico, y muy elevado $(\mathrm{p}<.0000)$, para las comparaciones entre los tres grupos de adolescentes.

En cuanto al conflicto entre el padre y la madre, y entre el hijo y ambos padres, de nuevo son los adolescentes delincuentes los que presentan los índices más altos de conflicto. Las mayores diferencias en el conflicto entre el padre y la madre se producen entre los no delincuentes y los delincuentes detectados $(p<.0000)$, son algo menores entre los no delincuentes y los delincuentes no detectados $(p<.0001)$, y menores aún, aunque elevados ( $<<.0002)$, entre ambos grupos de delincuentes. Con respecto al conflicto entre el hijo y los padres, las mayores diferencias se producen entre los no delincuentes y ambos grupos de delincuentes $(p<.0000)$ siendo un poco menor entre delincuentes detectados y no detectados $(p<.0002)$. $\mathrm{De}$ estos resultados en las variables referidas al conflicto, parece desprenderse que el conflicto en las relaciones familiares guarda una estrecha relación con la institucionalización del adolescente.

Con respecto a la calidad de las relaciones del hijo con cada uno de sus padres, se observa que existen diferencias significativas entre los grupos en cuanto a la relación con el padre, siendo los adolescentes delincuentes los que manifiestan una relación "menos buena" con su padre. La mayor diferencia se produce entre no delincuentes y delincuentes detectados $(p<0000)$, es menor entre no delincuentes y delincuentes detectados $(p<001)$, y menor aún entre ambos grupos de delincuentes $(p<.01)$. Sin embargo, no existen diferencias significativas entre los grupos en cuanto a la relación del hijo con la madre. No obstante, es importante destacar que, tanto los adolescentes delincuentes como los no delincuentes manifiestan 
una "mejor" relación con su madre que con su padre, siendo en los dos grupos de delincuentes donde se observan las mayores discrepancias entre la relación con el padre y con la madre.

Por último, en cuanto a las variables referidas a las relaciones normativas en el hogar (organización y control) se observa que el nivel de significación de las diferencias entre los grupos es claramente menor que para las variables afectivas.

La organización familiar, de acuerdo con la hipótesis formulada, es mayor para los no delincuentes que para ambos grupos de delincuentes. Esta variable establece diferencias especialmente importantes entre no delincuentes y delincuentes detectados $(\mathrm{p}<.0001)$ y algo menores entre no delincuentes y delincuentes no detectados $(p<.0009)$. Sin embargo, no existen diferencias significativas en esta variable entre ambos grupos de delincuentes.

El control familiar también establece diferencias entre delincuentes y no delincuentes, $y$, como se hipotetizaba, es mayor el control en las familias de delincuentes. Sin embargo, el nivel de significación de las diferencias para la comparación entre no delincuentes y cada uno de los grupos de delincuentes no es muy elevado $(p<.01)$. De nuevo, entre delincuentes detectados y no detectados las diferencias no son significativas.

La semejanza de los dos grupos de delincuentes en cuanto a estas dos variables parece sugerir que este podría ser un aspecto importante para establecer diferencias entre el ambiente familiar de no delincuentes y delincuentes (tanto detectados como no detectados).

Resumiendo los resultados del análisis de varianza podría establecerse que los adolescentes no delincuentes se diferencian de los dos grupos de adolescentes delincuentes en todas las variables utilizadas (con la excepción de la variable «relación del hijo con la madre»), mientras que los dos grupos de adolescentes delincuentes son similares en el nivel de confianza con sus padres y en la organización y el control familiar. Las principales diferencias entre ellos se establecen en cuanto al apego y a la alienación con los padres y, especialmente, en cuanto al conflicto en el hogar. Estos datos podrían estar indicando que el conflicto familiar y la ausencia de adecuadas relaciones entre el adolescente y sus padres se relacionan con una «ruptura" del sistema familiar, ruptura que incrementaría la probabilidad de que el adolescente fuese institucionalizado al realizar conductas delictivas.

En general, podría afirmarse, entonces, que el ambiente familiar de los adolescentes no delincuentes es significativamente diferente del de los delincuentes, mientras que el ambiente familiar de ambos tipos de delincuentes (detectados y no detectados) presenta un mayor grado de similitud.

Una vez que se ha comprobado que las variables familiares utilizadas, consideradas aisladamente, establecen diferencias entre delincuentes y no delincuentes, se ha realizado un análisis discriminante "paso a paso" (programa BMDP7M) con el fin de determinar la relevancia de las variables, consideradas conjuntamente, para discriminar entre los grupos de sujetos.

Tal como puede verse en la tabla IV, el análisis discriminante selecciona 4 variables: 1) apego entre el hijo y los padres $(F=161.12), 2)$ confianza entre el hijo y los padres $(F=22.25)$; 3 ) relación entre el hijo y la madre $(F=5,65)$, y 4$)$ conflicto familiar $(F=5.04)$. 


\section{6}

TABLA IV

Análisis discriminante por pasos: valor $F$ y peso de cada variable para definir la pertenencia al grupo

\begin{tabular}{rrrrrr}
\hline & & & \multicolumn{3}{c}{ Funciones discriminantes } \\
\cline { 4 - 6 } Pasos & \multirow{2}{*}{ Variables } & F & Grupo 1 & Grupo 2 & Grupo 3 \\
\hline \multirow{2}{nnnyyy}{1} & Apego & 161,12 &,- 08258 &,- 51932 &,- 84331 \\
2 & Confianza & 22,25 & 1,23020 & 1,61908 & 2,09186 \\
3 & Relación hijo-madre & 5,65 & 3,29753 & 4,15430 & 5,01570 \\
4 & Conflicto familiar & 5,04 & 2,42574 & 2,53885 & 3,05081 \\
& & Constante: & $-38,62918$ & $-33,18504$ & $-43,51028$ \\
\hline
\end{tabular}

Todas las variables seleccionadas hacen referencia a las relaciones afectivas familiares. Es importante señalar que el apego es la primera variable seleccionada, y con un valor $\mathrm{F}$ considerablemente mayor que el de las tres restantes. Otro resultado interesante es la presencia de la variable "relación del hijo con la madre» en los resultados del análisis. Es decir, aunque en los análisis previos se había observado que, considerada aisladamente, esta variable no establece diferencias entre delincuentes y no delincuentes, considerada conjuntamente con el resto de las variables se revela como un aspecto importante para discriminar entre los grupos de adolescentes.

$\mathrm{La}$ asignación de sujetos a grupos en base a sus puntuaciones en las variables que configuran las funciones de discriminación, muestra (tabla V) que estos grupos coinciden, en gran parte, con los establecidos «a priori» en este trabajo en función de sus autoinformes de conducta delictiva. Es decir, las variables seleccionadas podrían ser utilizadas como «predictores válidos" de la involucración del adolescente en actividades desviadas.

TABLA V

Número y porcentaje de sujetos clasificados correctamente en cada uno de los grupos

\begin{tabular}{lcccc}
\hline \multirow{2}{*}{, Grupo } & $\begin{array}{c}\text { Porcentaje de } \\
\text { casos clasificados } \\
\text { correctamente }\end{array}$ & \multicolumn{3}{c}{ Número de casos en cada grupo } \\
\cline { 3 - 5 } & Grupo 1 & Grupo 2 & Grupo 3 \\
\hline Grupo 1 & 86,0 & 37 & 6 & 0 \\
Grupo 2 & 76,6 & 2 & 36 & 9 \\
Grupo 3 & 86,0 & 0 & 6 & 36 \\
TOTAL: & 82,7 & 39 & 48 & 46 \\
\hline
\end{tabular}

En general, los resultados del análisis discriminante parecen confirmar que: 1) las variables afectivas se revelan como más importantes que las referidas a los aspectos normativos para establecer diferencias entre delincuentes y no delincuentes, y 2) el apego a los padres, junto con el conflicto en el hogar, parecen variables clave para establecer diferencias entre delincuentes y no delincuentes, asociándose un alto nivel de apego/bajo nivel de conflicto con no delincuencia, y un bajo nivel de apego/alto nivel de conflicto con delincuencia. 


\section{DISCUSION Y CONCLUSIONES}

De acuerdo con los presupuestos teóricos y los hallazgos empíricos previos, los resultados del presente estudio ponen de manifiesto que el ambiente familiar juega un papel fundamental en la conducta delictiva del adolescente.

Algunos de los estudios realizados anteriormente, habían trabajado únicamente con delincuencia detectada o con delincuencia no detectada; en este trabajo se han utilizado ambos tipos de delincuencia, y los resultados muestran que ambos grupos de sujetos presentan una cierta similitud en cuanto a las características de su ambiente familiar, y que este ambiente familiar es significativamente diferente del de los adolescentes no delincuentes.

Autores como Hirschi (1969), Netler (1974) y Reiss (1975) habían senalado que cuando se analizan variables psicosociales, tales como las del ambiente familiar, los hallazgos son similares tanto para delincuencia oficial como para delincuencia medida en base a autoinformes. Es decir, este tipo de variables psicosociales parecen estar relacionadas con conducta delictiva como tal, y no únicamente con institucionalización.

Sin embargo, y como ya se ha señalado, la institucionalización sí parece estar especialmente relacionada con algunos aspectos "negativos" del ambiente familiar, como el conflicto en el hogar o la alienación del adolescente con sus padres. Este dato puede ser interpretado en base a que las relaciones conflictivas entre los padres, y entre ambos padres y el hijo, pueden promover la ruptura del hogar (McCord, 1982), y es razonable suponer que un adolescente con conducta delictiva tiene más probabilidad de ser institucionalizado si procede de un hogar roto que si pertenece a una familia en la que ambos padres están presentes en el hogar y mantienen buenas relaciones.

En cuanto a la relevancia de las distintas variables utilizadas, de cara a establecer diferencias entre delincuentes y no delincuentes, los datos encontrados en este trabajo confirman, especialmente, el énfasis que las Teorías del Control conceden a la vinculación afectiva (apego) entre el hijo y los padres.

En las Teorías del Control, el apego es considerado como el principal mecanismo que actúa apartando al hijo de la realización de conducta delictiva (Nye, 1958; Hirschi, 1969). Del mismo modo, la falta de apego entre padres e hijos ha sido relacionada, en diversos estudios, con conducta delictiva del adolescente (Linden y Hackler, 1973; Cortés y Gatti, 1972; Norland y col., 1979).

En el presente estudio, el apego se revela como la variable fundamental para establecer diferencias entre los delincuentes y los no delincuentes. Los sujetos no delincuentes experimentan un grado de apego hacia sus padres significativamente mayor que los delincuentes. En este sentido, es importante señalar que el Inventario de Apego a los Padres (Greenberg y col., 1983; Armsden y Greenberg, en prensa) parece ser un instrumento especialmente idóneo para discriminar entre el nivel de apego a los padres de delincuentes y no delincuentes.

Además del apego, todas las variablés afectivas utilizadas en este trabajo establecen diferencias entre los grupos de adolescentes. 
Con respecto a la cohesión y al conflicto familiar, coincidiendo con las afirmaciones de Kogan (1980), se observa que las familias de los adolescentes delincuentes se caracterizan por un bajo nivel de cohesión y un alto nivel de conflicto.

En cuanto a las relaciones del hijo con cada uno de sus padres, aparecen resultados interesantes. Por una parte, las relaciones del hijo con el padre, tal como se sugería en estudios precedentes (Glueck y Glueck, 1950; McCord, 1983), parece jugar un papel importante en la delincuencia del adolescente. La relación con el padre puede ser especialmente relevante para los adolescentes varones, dado que una relación inadecuada o "negativa» puede llevar asociada una ausencia de modelos de rol (Gove y Crutchfield, 1982), con lo cual aumenta la probabilidad de que el adolescente busque esos modelos fuera de casa, incluso con compañeros no convencionales. $Y$ por lo que respecta a la relación del hijo con la madre, aunque en los primeros análisis parecía jugar un papel secundario, los resultados del análisis discriminante indica que es también una variable importante para discriminar entre delincuentes y no delincuentes. Este dato coincide, igualmente, con los de investigaciones previas (Glueck y Glueck, 1950; McCord, 1979; Olweus, 1980) en las cuales se enfatiza la importancia de una buena relación entre el hijo y la madre, tanto para un proceso adecuado de socialización, como para evitar la involucración del hijo en actividades delictivas.

En cuanto a las variables referidas a las relaciones normativas, aunque también aparecen como significativas para discriminar entre delincuentes y no delincuentes, su impacto en los resultados de este estudio es mucho menor que el de las variables afectivas. Estos resultados tal vez sean debidos a que en este trabajo se han analizado más a fondo los aspectos afectivos, introduciendo un mayor número de índices de relaciones afectivas. En todo caso, en posteriores investigaciones, sería necesario reexaminar este aspecto, introduciendo, además, algunas variables referidas a las técnicas o estilos educativos utilizados por los padres.

De todos modos, es interesante destacar que los resultados, en cuanto a la organización y al control familiar se producen en el sentido esperado y señalado en investigaciones previas. La Organización es mayor en las familias de no delincuentes y el control es significativamente mayor para los delincuentes. Estos datos están en la línea de las investigaciones que demuestran que tanto la ausencia de supervisión paterna como el exceso de control, en la adolescencia, pueden actuar promoviendo la conducta delictiva que, en principio, se pretende evitar (Conger, 1977; Rollins y Thomas, 1977; Wilson, 1980; Goldstein, 1984).

En general, puede afirmarse que los hallazgos del presente estudio confirman que determinadas características del ambiente familiar se relacionan con la delincuencia del adolescente, y que estas característias se refieren a un funcionamiento familiar y inadecuado.

Aunque es imposible, en un estudio transversal, establecer relaciones causales, la fuerza de algunos resultados encontrados, indica que esta es una línea de trabajo prometedora; y que es necesario profundizar en algunos aspectos, tales como, y por ejemplo, la clarificación del constructo apego a los padres, y de su relevancia en la conducta delictiva, dado que esta variable parece ser especialmente importante en la relación entre ambiente familiar y delincuencia juvenil. También sería interesante realizar estudios 
en los que se analicen exhaustivamente los aspectos referidos a las prácticas educativas y a todo el proceso de internalización de normas. Pero, y sobre todo, es necesaria la realización de trabajos longitudinales que permitan el establecimiento de relaciones de causalidad entre las variables familiares y la delincuencia del adolescente.

Este último tipo de estudios servirían de base para la elaboración y puesta en práctica de programas de prevención e intervención en el área de la delincuencia juvenil. Algunos programas de este tipo han sido, y están siendo, llevados a cabo por numerosos autores (Patterson y Fleischman, 1979; Denicola y Sandler, 1980; Alexander y Barton, 1980; Herz y col., 1984), y los primeros resultados indican que la intervención sobre los patrones de relación y las características del ambiente familiar, así como los programas de entrenamiento de los padres en técnicas educativas, pueden suponer soluciones al problema de la conducta delictiva de los adolescentes.

\section{Referencias}

AleXANDER, J. F., y BARTON, C. (1980). *Systems-Behavioral Intervention with Delinquent Families: Clinical, Metodological, and Conceptual Considerations». Advances in Family Intervention, Assessment and Theory, 1:53-87.

ARmsden, G. G., y Greenberg, M. T. *The Inventory of Parent and Peer Attachment: Individual Differences and their Relationship to Psychological Well-Being in Adolescence (en prensa).

BEBCHNUCK, J. (1983). «Family: A Relational Model». Acta Psiquiatrica y Psicológica de America Latina, 29:2, 138-145.

BowlBY, J. (1969). Attacbment and Loss. Vol. I: Attachment. Basic Books, New York.

Bowlby, J. (1973): Attachment and Loss. Vol. II: Separation. Basic Books, New York.

ChILTON, R. J., y MARKLE, G. E. (1972). «Family Disruption, Delinquent Conduct and the Effect of Subclassification*. American Sociological Review, 37:93-99.

CONGER, J. J. (1977). Adolescence and Youth: Psychological Development in a Changing World. New York: Harper \& Row.

CORTES, J. B., y GATTI, F. M. (1972). Delinquency and Crime: A Biopsychosocial Approach. New York: Seminar Press.

DAvIES, M., y SinClaIR, I. (1971). *Families, Hostels and Delinquents: An Attempt to Assess Cause and Effectw. The British Joumal of Criminology, 11:3, 213-229.

DENICOLA, J. y SANDLER, J. (1980). "Training of use of Parents in Child Management and Self Control Skillm. Behavior Therapy, 11, 263-270.

EMPEY, L. T., y LUBECK, S. G. (1971): Explaining Delinquency. Lexintong Mass: DC Healt.

FARRINGTON, D. P. (1973). «Self-Reports of Deviant Behavior: Predictive and Stable?». Journal of Criminal Law and Criminology, 64:99-110.

FARRINGTON, D. P. (1979). *Environmental Stress, Delinquent Behavior and Convictions*. En I. G. Saranson y C. D. Spielberg, Stress and Anxiety. New York: Wiley.

FisCHER, D. G. (1983). «Parental Supervision and Delinquency». Perceptual and Motor Skills, $56: 635-640$.

FISCHER, D. G. (1984). «Family Size and Delinquency*. Perceptual and Motor Skills, 58:2.

GlUECK, S., y GLUECK, E. (1950). Unraveling Juvenile Delinquency. Cambridge, Mass: Harvard University Press.

GluECK, S., y GLUECK, E. (1968). Delinquents and Non-Delinquents in perspective. Cambridge: Harvard University Press.

GLUECK, S., y GLUECK, E. (1970). Toward a Typology of Juvenile Offenders: Implications for Therapy and Prevention. New York: Grune and Stratton.

Goldstein, H. S. (1984). *Parental Composition, Supervision, and Conduct Problems in Youths 12 to 17 Years Old m. Joumal of the American Academy of Child Psychiatry, 23:6, 679-685.

GOVE, W. R., y CRUTCHFIELD, R. D. (1982). «The Family and Juvenile Delinquency». The Sociological Quarterly, 23:301-319.

GREenberG, M. T.; SIEgEL, J. M., y LeitCh, C. (1983). *The Nature and Importance of Attachment Relationships to Parents and Peers During Adolescence». Journal of Youth and Adolescence, 12:5, 373-387. 
Hason, C. L.; Henggeler, S. W.; Haefele, W. F., y RodiCK, J. D. (1984). «Demographic, Individual, and Family Relations Correlates of Serious and Repeated Crime Among Adolescents and Their Sibblings». Joumal of Consulting and Clinical Psychology, 52, 4, 528-539.

HARBIN, M. T., y MADDEN, D. J. (1982). *Assaultive Adolescents: Family Decision Making Parameters». Family Process, 22:109-118.

HiRSCHI, T. (1969). Causes of Delinquency. Berkely: University of California Press.

HERZ, E. J.; GolDberG, W. A., y REIS, J. S. (1984). «Family Life Education for Young Adolescents: A Quasi-Experiment». Journal of Youth and Adolescence, 13:4, 309-329.

KogAN, L. (1980). "A Family Systems Perspective on Status Offenders». Journal of Juvenile and Family Courts, 31:2.

LINDEN, R., y HACKLER, J. (1973). «Affective Ties and Delinquency". Pacific Sociological Review, 16:27-46.

LINDEN, R., y Fillmore, C. (1981). "A Comparative Study of Delinquency Involvement". Canadian Review of Sociology and Anthropology, 18:3.

LOEBER, R., y DiSHION, T. J. (1984). «Boys who Fight at Home and School: Family Conditions Influencing Cross-Setting Consistency". Journal of Consulting and Clinical Psycho$\log y, 52: 5$.

Mateo, M. A. (1982). Cuestionario C.T.A. y Conducta Antisocial. Memoria de Licenciatura. Universidad Complutense. Madrid.

MCCORD, J. A. (1979). «Some Child-Rearing Antecedents of Criminal Behavior in Adult Men». Journal of Personality and Social Psychology, 37:9, 1477-1486.

MCCORD, J. A. (1982). "A Longitudinal Wiew of the Relationship between Paternal Absence and Crime». En J. Gunn y D. Farrington (Eds). Abnormal Offenders, Delinquency, and the Criminal Justice System. New York: Wiley.

MCCORD, J. A. (1983). «Family Relationships and Crime». En Encyclopedia of Crime and Justice. S. H. Dadish (Ed.). New York: Free Press.

Moos, R. H., y Moos, P. S. (1976). «A Typology of Family Social Environments». Family Process, 15:357-371.

Moos, R. H., Moos, P. S. (1981). Family Environment Scale. Manual. Consulting Psychologist Press. Palo Alto, C. A.

NetTler, G. (1974). Explaining Crime. New York: McGraw Hill.

Norland, S.; SHOVER, N.; THORNTON, W., y JAMES, J. (1979). «Intrafamily Conflict and Delinquency». Pacific Sociological Review, 22:223-240.

MúÑEZ GARCfA, M. (1983). Personalidad, Lugar de Control y sus Relaciones con la Conducta Antisocial. Memoria de Licentuatura. Universidad de Santiago.

NYE, F. I. (1957). «Child Adjustement in Broken and in Unhappy Unbroken Homes». Marriage and Family Living, 19:356-361.

NYE, F. I. (1958). Family Relationships and Delinquent Bebavior. New York: John Wiley and Sons.

OFFORD, D. R. (1982). «Family Background of Male and Female Delinquents». En Abnormal Offenders, Delinquency and the Criminal Justice Systems, J. Gunn \& D. P. Farrington (Ed.). Chichester: Wiley.

OLwEUS, D. (1980). "Familial and Temperamental Determinants of Aggresive Behavior in Adolescent Boys: A Causal Analysis». Development Psychology, 16:644-660.

PATTERSON, G. R., y FleisCHMAN, M. J. (1979). "Maintenance of Treatment Effects: Some Considerations Concerning Family Systems and Follow-up Datax. Behavior Therapy, 10:168-195.

REISS, D. (1971). *Varieties of Consensual Experience III: Contracts between Families of Normals, Delinquents and Schizophrenics». Journal Nerv. Ment. Dis., 152:73-95.

REISS, A. (1975), «Inappropiate Theories and Inadequate Methods as Policy Plagres: Self-Reported Delinquency and the Law». En N. J. Demerath, O. L. Larsen y K. Schuessler (Eds.). Social Policy and Sociology. New York: Academic Press.

Rollins, B. C., y Thomas, D. L. (1975). «A Theory of Parental Power and Child Compilance». En R. C. Cromwell y D. H. Dison (Eds.). Power in Families. New York. Holsted and John Wiley.

RUTTER, M. (1971). «Parent-Child Separation: Psychological Effects on the Children».Journal of Child Psychology and Psychiatry, 12:233-260.

STANFIELD, R. (1966). *The Interaction of Family Variables and Gang Variables n the Etiology of Delinquecy». Social Problems, 13.

SPICER, J. W., y HAMPE, G. D. (1975). "Kinship Interaction after Divorce . Journal of Marriage and the Family, 37:113-119.

WEST, D. J. (1969). Present Conduct and Future Delinquency. London. Heinemann.

WEST, D. J., y FARRINGTON, D. P. (1977). The Delinquent Way of Life. London: Heinemann.

WILSON, H. (1980). "Parental Supervision: A Neglected Aspect of Delinquency *. British Journal of Criminology, 20, 203-235.

WRIGHT, L. S. (1982). *Parental Permision to Date and its Relationship to Drug Use and Suicidal Thoughts among Adolescentsm. Adolescence, vol. 7:66, 409-419. 